\title{
Weighted Estimates for Oscillatory Singular Integrals
}

\author{
H. Al-Qassem, ${ }^{1}$ L. Cheng, ${ }^{2}$ A. Fukui, ${ }^{2}$ and Y. Pan ${ }^{3}$ \\ ${ }^{1}$ Department of Mathematics and Physics, Qatar University, Doha, Qatar \\ ${ }^{2}$ Department of Mathematics, Bryn Mawr College, Bryn Mawr, PA 19010, USA \\ ${ }^{3}$ Department of Mathematics, University of Pittsburgh, Pittsburgh, PA 15260, USA \\ Correspondence should be addressed to Y. Pan; yibiao@pitt.edu
}

Received 25 January 2013; Accepted 15 March 2013

Academic Editor: Alberto Fiorenza

Copyright (C) $2013 \mathrm{H}$. Al-Qassem et al. This is an open access article distributed under the Creative Commons Attribution License, which permits unrestricted use, distribution, and reproduction in any medium, provided the original work is properly cited.

\begin{abstract}
We establish uniform bounds for oscillatory singular integrals as well as oscillatory singular integral operators. We allow the singular kernel to be given by a function in the Hardy space $H^{1}\left(\mathbf{S}^{n-1}\right)$, while such results were known previously only for kernels in $L$ log $L\left(\mathbf{S}^{n-1}\right)$, a proper subspace of $H^{1}\left(\mathbf{S}^{n-1}\right)$. One of our results established a $L^{p}(w) \rightarrow L^{p}(w)$ bound for certain weights. At the same time, it provides a solution to an open problem in $\mathrm{Lu}(2005)$.
\end{abstract}

\section{Introduction}

In this paper we establish uniform bounds for oscillatory singular integrals. We consider two types of oscillatory singular integrals, which will be described later.

Let $n \geq 2$ and $\mathbf{S}^{n-1}$ denote the unit sphere in $\mathbf{R}^{n}$ equipped with the induced Lebesgue measure $\sigma$. For an integrable function $\Omega: S^{n-1} \rightarrow$ C satisfying

$$
\int_{S^{n-1}} \Omega d \sigma=0,
$$

we define

$$
K(x)=\frac{\Omega\left(x^{\prime}\right)}{|x|^{n}}
$$

where $x^{\prime}=x /|x|$ for $x \in \mathbf{R}^{n} \backslash\{0\}$. For $d, m \in \mathbf{N}$, let

$$
\begin{aligned}
\mathscr{P} & (d, m) \\
& =\left\{P: \mathbf{R}^{m} \longrightarrow \mathbf{R}: P \text { be a polynomial with } \operatorname{deg}(P) \leq d\right\} .
\end{aligned}
$$

Type I. An oscillatory integral of type I is given by

$$
I_{n}(\Omega, P)=\text { p.v. } \int_{\mathbf{R}^{n}} e^{i P(x)} K(x) d x,
$$

where $K$ is given by (2) and $P$ is a polynomial on $\mathbf{R}^{n}$. For a given $\Omega: \mathbf{S}^{n-1} \rightarrow \mathbf{C}$ and $d \in \mathbf{N}$ the main concern is to establish a bound for

$$
\sup _{P \in \mathscr{P}(d, n)}\left|I_{n}(\Omega, P)\right|
$$

Previous results in this regard include Stein [1] for $\Omega \epsilon$ $L^{\infty}\left(\mathbf{S}^{n-1}\right)$ and Papadimitrakis and Parissis [2] for $\Omega \in$ $L \log L\left(\mathbf{S}^{n-1}\right)$ which improved Stein's result.

Type II. A type II oscillatory singular integral is actually an integral operator of the form

$$
T_{\Omega, \mathrm{Q}}: f \longrightarrow \text { p.v. } \int_{\mathbf{R}^{n}} e^{i \mathrm{Q}(x, y)} K(x-y) f(y) d y,
$$

where $K$ is given by (2) and $Q$ is a real-valued polynomial on $\mathbf{R}^{n} \times \mathbf{R}^{n}$. Ricci and Stein [3] showed that, if $\Omega \in C^{1}\left(\mathbf{S}^{n-1}\right)$, $T_{\Omega, Q}$ is bounded on $L^{p}\left(\mathbf{R}^{n}\right)$. Subsequently $L u$ and Zhang [4] and Jiang and $\mathrm{Lu}$ [5] established the same bounds for $\left\|T_{\Omega, Q}\right\|_{p, p}$ under the weaker conditions $\Omega \in L^{1+\epsilon}\left(\mathbf{S}^{n-1}\right)$ and $\Omega \in L \log L\left(\mathbf{S}^{n-1}\right)$, respectively.

We will now state our main results, beginning with oscillatory singular integrals of Type II. 
A set $R$ in $\mathbf{R}^{n}$ is called a rectangle if there is an orthonormal basis $\left\{e_{1}, \ldots, e_{n}\right\}$ of $\mathbf{R}^{n}$ (which may depend on $R$ ) such that

$$
R=\left\{\sum_{j=1}^{n} x_{j} e_{j}: a_{j} \leq x_{j} \leq b_{j}, \text { for } 1 \leq j \leq n\right\} .
$$

In other words, what we call a rectangle in $\mathbf{R}^{n}$ is simply any rotation of an arbitrary $n$-cell $\left[a_{1}, b_{1}\right] \times \cdots \times\left[a_{n}, b_{n}\right]$. Let $\mathscr{R}_{n}$ denote the collection of all rectangles in $\mathbf{R}^{n}$.

Definition 1. Let $p \in(1, \infty)$, and let $w$ be a nonnegative, locally integrable function on $\mathbf{R}^{n}$. We say that $w$ is in the weight class $V_{p}$ if

$$
\sup _{R \in \mathscr{R}_{n}}\left(\frac{1}{|R|} \int_{R} w(x) d x\right)\left(\frac{1}{|R|} \int_{R} w(x)^{-1 /(p-1)} d x\right)^{p-1}<\infty .
$$

It is easy to see that $V_{p}$ is a subcollection of the well-known weight class $A_{p}$ of Muckenhoupt $[6,7]$. Examples of weights in $V_{p}$ include all weights of the form $|G(x)|^{\alpha}$, where $G(x)$ is a polynomial in $\mathbf{R}^{n}$ and $-1<\alpha \operatorname{deg}(G)<p-1$.

Theorem 2. Let $Q(x, y)$ be a real-valued polynomial on $\mathbf{R}^{n} \times$ $\mathbf{R}^{n}$. Suppose that $w \in V_{p}, \Omega \in H^{1}\left(\mathbf{S}^{n-1}\right)$ and $\Omega$ satisfies (1). Then the operator $T_{\Omega, Q}$ is bounded on $L^{p}\left(\mathbf{R}^{n}, w\right)$ for $1<p<$ $\infty$, with a bound on its norm which may depend on the degree of $Q$ but is otherwise independent of the coefficients of $Q$.

The space $H^{1}\left(\mathbf{S}^{n-1}\right)$ is the Hardy space on the unit sphere. Since $L \log L\left(\mathbf{S}^{n-1}\right)$ is a proper subspace of $H^{1}\left(\mathbf{S}^{n-1}\right)$, Theorem 2 represents an improvement over results mentioned earlier. By taking $w=1$, it answers an open question in [8, page 52] in the affirmative.

Our second result has the same flavor as the first, but it concerns Type I oscillatory singular integrals instead.

Theorem 3. Suppose that $\Omega \in H^{1}\left(\mathbf{S}^{n-1}\right)$ and $\Omega$ satisfies (1). Then

$$
\sup _{P \in \mathscr{P}(d, n)}\left|I_{n}(\Omega, P)\right| \leq c_{n}(1+\log d)\|\Omega\|_{H^{1}\left(\mathbf{S}^{n-1}\right)},
$$

where $c_{n}$ is a constant independent of $d$ and $\Omega$.

Our result in this regard is built on the work of Papadimitrakis and Parissis who gave the following bound in [2]:

$$
\sup _{P \in \mathscr{P}(d, n)}\left|I_{n}(\Omega, P)\right| \leq c(1+\log d)\left(\|\Omega\|_{L \log L\left(\mathbf{S}^{n-1}\right)}+1\right) .
$$

They also showed the logarithmic growth of the bound in $d$ to be best possible. Our bound, while dependent on the dimension $n$, provides an improvement over the factor $\left(\|\Omega\|_{L \log L}+1\right)$.

\section{Proofs of Theorems 2 and 3}

We will begin by recalling the atomic decomposition for $H^{1}\left(\mathbf{S}^{n-1}\right)$.
Definition 4. A measurable function $a(\cdot)$ on $S^{n-1}$ is called a regular $H^{1}$ atom if it satisfies the following:

(i) $\int_{\mathbf{S}^{n-1}} a(y) d \sigma(y)=0$,

(ii) $\operatorname{supp}(a) \subseteq \mathbf{S}^{n-1} \cap B\left(\theta_{0}, \rho\right)$ for some $\theta_{0} \in \mathbf{S}^{n-1}$ and $\rho>0$, where $B\left(\theta_{0}, \rho\right)=\left\{y \in \mathbf{R}^{n}:\left|y-\theta_{0}\right|<\rho\right\}$,

(iii) $\|a\|_{\infty} \leq \rho^{-n+1}$.

An exceptional atom is just an $L^{\infty}$ function $a(\cdot)$ on $S^{n-1}$ satisfying $\|a\|_{\infty} \leq 1$.

The following result is from $[9,10]$.

Lemma 5. For every $h \in H^{1}\left(\mathbf{S}^{n-1}\right)$ there exist $\left\{\lambda_{k}\right\} \subset \mathbf{C}$ and $H^{1}$ atoms (both regular and exceptional) $\left\{a_{k}(\cdot)\right\}$ such that

$$
h=\sum_{k} \lambda_{k} a_{k}
$$

and $\|h\|_{H^{1}\left(\mathbf{S}^{n-1}\right)} \approx \sum_{k}\left|\lambda_{k}\right|$.

Proof of Theorem 2. Let $d=\operatorname{deg}(Q)$. It suffices to show that, for $1<p<\infty$, there exists a $C(n, d, p, w)>0$ such that

$$
\left\|T_{\Omega, Q} f\right\|_{L^{p}\left(\mathbf{R}^{n}, w\right)} \leq C(n, d, p, w)\|\Omega\|_{H^{1}\left(\mathbf{S}^{n-1}\right)}\|f\|_{L^{p}\left(\mathbf{R}^{n}, w\right)},
$$

for all $f \in C_{0}^{\infty}\left(\mathbf{R}^{n}\right)$. Since the sum in (11) converges in the sense of distribution, by Lemma 5 , we only need to prove

$$
\left\|T_{\Omega, Q}\right\|_{L^{p}\left(\mathbf{R}^{n}, w\right) \rightarrow L^{p}\left(\mathbf{R}^{n}, w\right)} \leq C(n, d, p, w),
$$

when $\Omega$ is a regular atom.

Below we will assume that $\Omega(\cdot)=a(\cdot)$ satisfies Conditions (i)-(iii) in Definition 4. Obviously we may also assume that $\rho \in(0,1 / 4)$. We also extend $\Omega$ to be a homogeneous function of degree 0 by setting $\Omega(x)=\Omega(x /|x|)$ for $x \in \mathbf{R}^{n} \backslash\{0\}$. Let $M$ be an $n \times n$ orthogonal matrix such that $\theta_{0} M^{t}=(0, \ldots, 0,1)=$ e. We define the linear transformation $\Gamma$ on $\mathbf{R}^{n}$ by

$$
\Gamma y=y\left(\begin{array}{cc}
\rho I_{n-1} & 0 \\
0 & 1
\end{array}\right) M
$$

where $I_{n-1}$ denotes the $(n-1) \times(n-1)$ identity matrix and $y=\left(y_{1}, \ldots, y_{n}\right)=\left(\tilde{y}, y_{n}\right)$. By letting $\Psi(x, y)=Q(\Gamma x, \Gamma y)$, $f_{\Gamma}(x)=f(\Gamma x), w_{\Gamma}(x)=\rho^{n-1} w(\Gamma x)$, and

$$
h(x)=\rho^{n-1}\left(\frac{|x|^{n} \Omega(\Gamma x)}{|\Gamma x|^{n}}\right),
$$

we get

$$
\left(T_{\Omega, \mathrm{Q}} f\right)(\Gamma x)=\left(T_{h, \Psi} f_{\Gamma}\right)(x) .
$$

If $h(x) \neq 0$ for some $x=\left(\tilde{x}, x_{n}\right) \in \mathbf{R}^{n} \backslash\{0\}$, then by (i) we have

$$
\left|\frac{\left(\rho \tilde{x}, x_{n}\right)}{\left|\left(\rho \tilde{x}, x_{n}\right)\right|}-\mathbf{e}\right|<\rho,
$$

which implies that

$$
x_{n}>\left(1-\frac{\rho^{2}}{2}\right)\left|\left(\rho \tilde{x}, x_{n}\right)\right| .
$$


Thus,

$$
\begin{aligned}
|x| & =\rho^{-1}\left(\left|\left(\rho \tilde{x}, x_{n}\right)\right|^{2}+\left(\rho^{2}-1\right) x_{n}^{2}\right)^{1 / 2} \\
& <\rho^{-1}\left[1+\left(\rho^{2}-1\right)\left(\frac{1-\rho^{2}}{2}\right)^{2}\right]^{1 / 2} \\
& \times\left|\left(\rho \tilde{x}, x_{n}\right)\right| \leq \sqrt{2}|\Gamma x| .
\end{aligned}
$$

Therefore we have

$$
\|h\|_{\infty} \leq 2^{n / 2}
$$

By its definition and a well-known argument, $h$ is homogeneous of degree 0 and satisfies (1). Also observe that $\operatorname{deg}(\Psi)=\operatorname{deg}(Q)$ and $w_{\Gamma}$ is an $A_{p}$ weight with an $A_{p}$ bound independent of $\Gamma$. Thus, by Theorem 5 in [11] and Theorem 5 of [5], there is a $C(n, d, p, w)>0$ such that

$$
\begin{aligned}
\left\|T_{\Omega, Q}\right\|_{L^{p}\left(\mathbf{R}^{n}, w\right) \rightarrow L^{p}\left(\mathbf{R}^{n}, w\right)} & =\left\|T_{h, \Psi}\right\|_{L^{p}\left(\mathbf{R}^{n}, w_{\Gamma}\right) \rightarrow L^{p}\left(\mathbf{R}^{n}, w_{\Gamma}\right)} \\
& \leq C(n, d, p, w) .
\end{aligned}
$$

This proves Theorem 2 .

Proof of Theorem 3. Let $P \in \mathscr{P}(d, n)$, and let $Q(x, y)=P(x-$ $y)$. For a $\Omega \in H^{1}\left(\mathbf{S}^{n-1}\right)$ which satisfies (1), we write

$$
\Omega=\sum_{j} \lambda_{j} a_{j}
$$

where $\left\{a_{j}\right\}$ are regular $H^{1}$ atoms and $\|\Omega\|_{H^{1}} \approx \sum_{j}\left|\lambda_{j}\right|$. By the proof of Theorem 2 , for each $j$, there exist a $P_{j} \in \mathscr{P}(d, n)$ and a function $\omega_{j}$ on $S^{n-1}$ which satisfies (1) and $\left\|\omega_{j}\right\|_{\infty} \leq 2^{n / 2}$ such that

$$
\left\|T_{\Omega, Q}\right\|_{2,2} \leq \sum_{j}\left|\lambda_{j}\right|\left\|T_{\omega_{j}, Q_{j}}\right\|_{2,2},
$$

where $Q_{j}(x, y)=P_{j}(x-y)$. By (23) and Theorem 1 in [2], we have

$$
\begin{aligned}
\left|I_{n}(\Omega, P)\right| & \leq\left\|T_{\Omega, Q}\right\|_{2,2} \leq \sum_{j}\left|\lambda_{j}\right|\left\|T_{\omega_{j}, Q_{j}}\right\|_{2,2} \\
& \leq c(1+\log d)\left(\sum_{j}\left|\lambda_{j}\right|\left\|\omega_{j}\right\|_{L \log L\left(\mathbf{S}^{n-1}\right)}\right) \\
& \leq c_{n}(1+\log d)\|\Omega\|_{H^{1}\left(\mathbf{S}^{n-1}\right)},
\end{aligned}
$$

which proves Theorem 3 .

\section{References}

[1] E. M. Stein, "Oscillatory integrals in Fourier analysis," in Beijing Lectures in Harmonic Analysis, vol. 112 of Annals of Mathematics Studies, pp. 307-355, Princeton University Press, 1986.

[2] M. Papadimitrakis and I. R. Parissis, "Singular oscillatory integrals on $R^{n}$," Mathematische Zeitschrift, vol. 266, no. 1, pp. 169-179, 2010.
[3] F. Ricci and E. M. Stein, "Harmonic analysis on nilpotent groups and singular integrals. I. Oscillatory integrals," Journal of Functional Analysis, vol. 73, no. 1, pp. 179-194, 1987.

[4] S. Z. Lu and Y. Zhang, "Criterion on $L^{p}$-boundedness for a class of oscillatory singular integrals with rough kernels," Revista Matemática Iberoamericana, vol. 8, no. 2, pp. 201-220, 1992.

[5] Y. S. Jiang and S. Z. Lu, "Oscillatory singular integrals with rough kernel," in Harmonic Analysis in China, vol. 327, pp. 135145, Kluwer Academic Publishers, 1995.

[6] B. Muckenhoupt, "Weighted norm inequalities for the Hardy maximal function," Transactions of the American Mathematical Society, vol. 165, pp. 207-226, 1972.

[7] R. R. Coifman and C. Fefferman, "Weighted norm inequalities for maximal functions and singular integrals," Studia Mathematica, vol. 51, pp. 241-250, 1974.

[8] S. Lu, "A class of oscillatory singular integrals," International Journal of Applied Mathematical Sciences, vol. 2, no. 1, pp. 4764, 2005.

[9] R. R. Coifman and G. Weiss, "Extensions of Hardy spaces and their use in analysis," Bulletin of the American Mathematical Society, vol. 83, no. 4, pp. 569-645, 1977.

[10] L. Colzani, Hardy spaces on spheres [Ph.D. thesis], Washington University, St. Louis, Mo, USA, 1982.

[11] J. Duoandikoetxea, "Weighted norm inequalities for homogeneous singular integrals," Transactions of the American Mathematical Society, vol. 336, no. 2, pp. 869-880, 1993. 


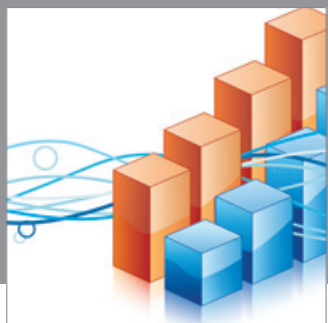

Advances in

Operations Research

mansans

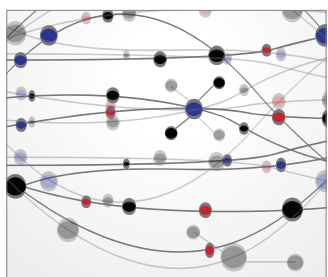

The Scientific World Journal
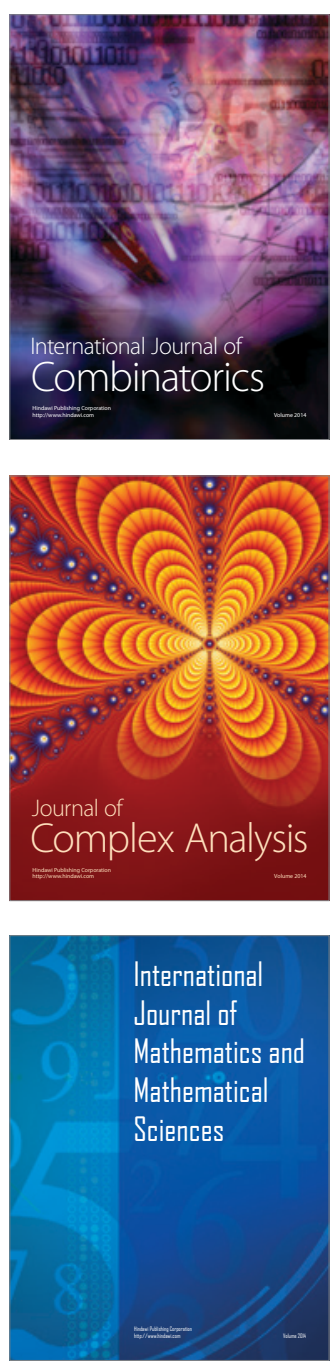
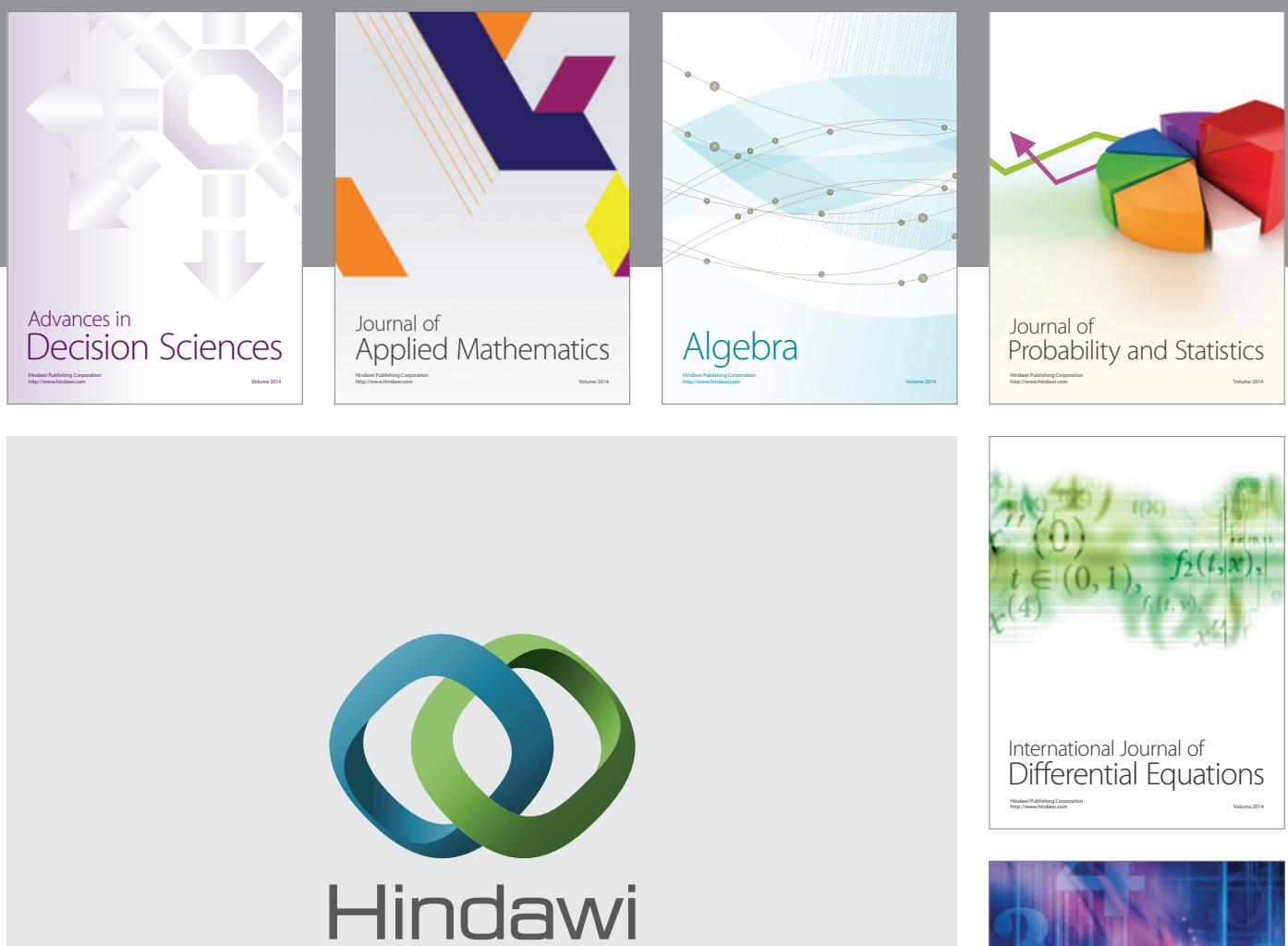

Submit your manuscripts at http://www.hindawi.com
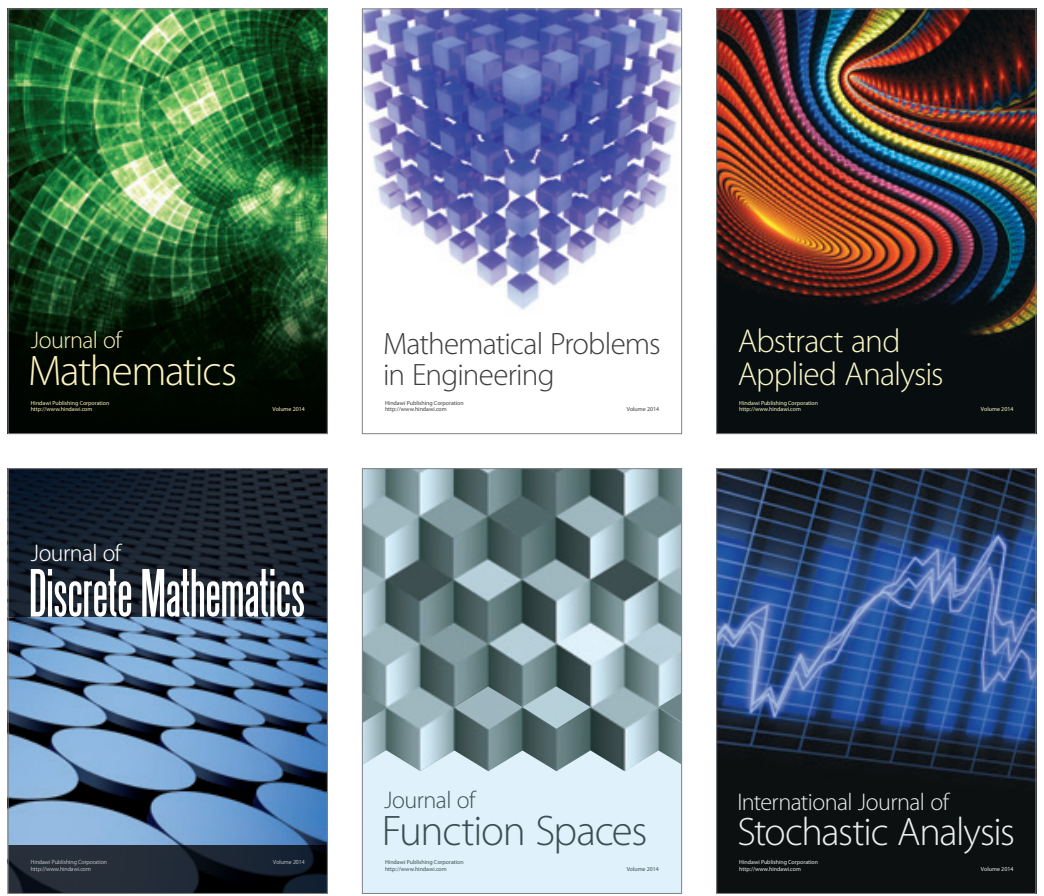

Journal of

Function Spaces

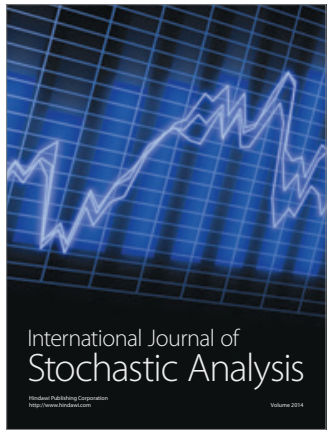

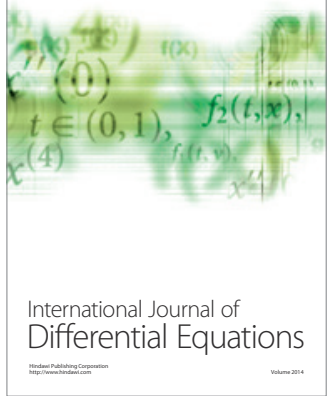
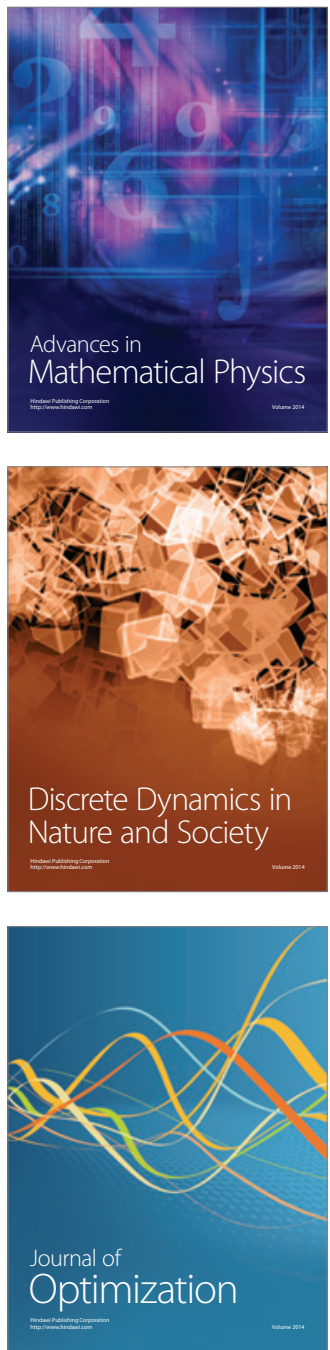